\title{
Treatment disparities and effect on late mortality in patients with diabetes presenting with acute myocardial infarction: observations from the ACACIA registry
}

\author{
Joseph Hung, David B Brieger, John V Amerena, Steven G Coverdale, James M Rankin, \\ Carolyn M Astley, Ashish Soman and Derek P Chew
}

$\mathrm{P}$ atients with diabetes have higher short-term and long-term mortality and morbidity rates after acute myocardial infarction (MI) than non-diabetic patients. ${ }^{1-7}$ Proven risk-modifying treatments have shown equivalent or larger relative risk reductions in diabetic compared with non-diabetic patients with acute coronary syndrome (ACS) ${ }^{8-10}$ The absolute benefit of these treatments among diabetic patients with ACS is potentially greater owing to their higher baseline risk. This underscores the importance of physician adherence to evidence-based guidelines in the treatment of patients with diabetes who present with ACS. However, data from several international registries suggest that disparities in mortality rates and treatments have persisted among diabetic compared with non-diabetic patients following acute MI, despite broad acceptance of ACS treatment guidelines. ${ }^{3-7}$

We used data from a prospective national registry of patients with ACS to compare the use of evidence-based pharmacological and invasive treatments and 12-month mortality rates between patients with and without diabetes who present with acute MI. We also explored the relationship between these treatments and 12-month clinical outcomes.

\section{METHODS}

The Acute Coronary Syndrome Prospective Audit (ACACIA, sanofi-aventis study protocol number PML-0051) has been described previously. ${ }^{11,12}$ The trial was conducted between 1 November 2005 and 31 July 2007 and involved 39 hospitals across all states and territories of Australia. Hospitals were selected to be representative of rural (25\%) and metropolitan (75\%) centres, and of interventional (83\%) and non-interventional $(17 \%)$ centres. ${ }^{11}$ The trial enrolled 3402 patients with suspected ST segment elevation MI (STEMI) or high-risk and intermediate-risk non-ST segment elevation ACS. ${ }^{13}$ This analysis was limited to the 1744 patients with a final discharge diagnosis of acute MI, determined by investigators at

\section{ABSTRACT}

Objectives: To compare the use of evidence-based pharmacological and invasive treatments and 12-month mortality rates between patients with and without diabetes who present with acute myocardial infarction (MI), and to explore the relationship between these treatments and late clinical outcomes.

Design and setting: Prospective, nationwide multicentre registry: the Acute Coronary Syndrome Prospective Audit (ACACIA).

Patients: Patients presenting to 24 metropolitan and 15 non-metropolitan hospitals with acute coronary syndrome (ACS) and a final discharge diagnosis of acute MI between November 2005 and July 2007.

Main outcome measure: All-cause mortality at 12 months.

Results: Nearly a quarter of 1744 patients with a final diagnosis of acute MI had a history of diabetes on presentation. Patients with diabetes were older, with a greater prevalence of comorbidities than non-diabetic patients, and were less likely to be treated at discharge with evidence-based medications (aspirin, clopidogrel, a statin and/or a $\beta$-blocker) or to receive early invasive procedures. After adjusting for baseline characteristics and therapeutic interventions, diabetes at presentation was independently associated with a higher mortality at 12 months after MI (hazard ratio, $1.79 ; 95 \% \mathrm{Cl}, 1.18-2.72 ; P=0.007)$. Early invasive management and discharge prescription of guideline-recommended medications were associated with a significantly reduced hazard of mortality at 12 months.

Conclusion: Patients with diabetes have a higher risk than non-diabetic patients of late mortality following an acute $\mathrm{Ml}$, yet receive fewer guideline-recommended medications and early invasive procedures. Increased application of proven pharmacotherapies and an early invasive management strategy in patients with diabetes presenting with ACS might improve their outcomes.

Study protocol number (sanofi-aventis): PML-0051.

MJA 2009; 191: 539-543

each hospital site and confirmed by a centralised query process. ${ }^{11}$

The trial was approved by the institutional ethics committee at each site. All patients included in the trial provided informed consent with the exception of those who died before consent was sought; access to their medical records was approved by the local ethics committees.

\section{Clinical and treatment variables}

Data were obtained from hospital medical records on demographic, clinical and procedural factors involved in the management of patients with ACS. Diabetes status at admission was based on a self-reported physician diagnosis and was further classi- fied by treatment type as diet only, oral hypoglycaemic agent(s), or needing insulin therapy. Those who were insulin-dependent included patients with type 1 diabetes. The estimated glomerular filtration rate (eGFR) was obtained from serum creatinine measurements and the Modification of Diet in Renal Disease formula. ${ }^{14}$ The Global Registry of Acute Coronary Events (GRACE) risk score containing relevant prognostic factors was derived for each patient. ${ }^{15} \mathrm{~A}$ discharge prescription of aspirin, clopidogrel, a statin, $\beta$-blocker, angiotensin-converting enzyme (ACE) inhibitor or angiotensin-receptor (AR) antagonist was recorded. Early invasive management was defined as angiography at any time during the acute hospital 
stay, regardless of transfer between acute hospitals. The use of percutaneous coronary intervention (PCI) and coronary artery bypass grafting (CABG) was also recorded. All data were collected by trained clinical coordinators.

All-cause mortality and time of death were determined to 12 months after the index hospitalisation. Vital status at 12 months could be confirmed in $99.9 \%$ (1741) of the cohort. Data on late non-fatal recurrent MI (re-MI), stroke and coronary revascularisation were obtained from hospital discharge summaries and diagnosisrelated group (DRG) coding reports.

\section{Statistical analysis}

Normally distributed variables were expressed as mean (SD), variables with a skewed distribution as median (interquartile range $[\mathrm{IQR}]$ ), and counts as number and percentage. Kaplan-Meier survival curves, stratified by diabetes treatment groups, were plotted and compared with the log-rank test.

The association between admission diabetes status and all-cause mortality and combined death, non-fatal re-MI or stroke at 12 months was assessed through Cox proportional hazards modelling, using robust sandwich standard error estimates to adjust for within-hospital clustering effects. The model was also adjusted for other significant clinical covariates, including age, prior MI, Killip Class, GRACE risk score, impaired renal function $\left(e G F R<60 \mathrm{~mL} / \mathrm{min} / 1.73 \mathrm{~m}^{2}\right.$ ), need for dialysis, early invasive management, and discharge treatment with aspirin, clopidogrel, a statin, $\beta$-blocker or ACEinhibitor/AR-antagonist. Diabetes status was entered into the model either as a binary variable or by category according to type of diabetes treatment.

As no interactions were observed between variables — including between diabetes status and rural/metropolitan hospital source - the simple models are presented. The proportional hazards ratio (HR) was assessed for each covariate. A probability $<0.05$ was considered statistically significant. All analyses were performed with Stata software, version 9.1 (StataCorp, College Station, Tex, USA).

\section{RESULTS}

Of the 1744 patients in the analysis, $24.3 \%$ (423) had a history of diabetes; about a third of these were treated with diet alone, a quarter required insulin therapy, and the remainder were taking oral hypoglycaemic

\begin{tabular}{|c|c|c|}
\hline Characteristic & No diabetes $(n=1321)$ & Diabetes $(n=423)$ \\
\hline Mean age (years) (SD) & $64.6(13.6)$ & $66.4(11.9)$ \\
\hline Male sex & $936(70.9 \%)$ & $292(69.0 \%)$ \\
\hline Hypertension & 706 (53.4\%) & $326(77.1 \%)$ \\
\hline Dyslipidaemia & $640(48.4 \%)$ & $282(66.9 \%)$ \\
\hline Current smoking & $385(29.1 \%)$ & $89(21.0 \%)$ \\
\hline \multicolumn{3}{|l|}{ Diabetes treatment } \\
\hline Diet only & na & $135(31.9 \%)$ \\
\hline Oral hypoglycaemic agent(s) & na & $187(44.2 \%)$ \\
\hline Insulin & na & $101(23.9 \%)$ \\
\hline Family history of coronary artery disease & $444(33.6 \%)$ & $118(27.9 \%)$ \\
\hline Prior myocardial infarction & $271(20.5 \%)$ & $114(26.9 \%)$ \\
\hline Prior percutaneous coronary intervention & $151(11.4 \%)$ & $69(16.3 \%)$ \\
\hline Prior coronary artery bypass grafting & $115(8.7 \%)$ & $67(18.8 \%)$ \\
\hline Prior atrial fibrillation & $104(7.9 \%)$ & $57(13.5 \%)$ \\
\hline Prior cerebrovascular accident & $60(4.5 \%)$ & $38(9.0 \%)$ \\
\hline Prior peripheral vascular disease & $62(4.7 \%)$ & $45(10.6 \%)$ \\
\hline \multicolumn{3}{|l|}{ Admission diagnosis } \\
\hline Acute STEMI & $592(44.8 \%)$ & $133(31.4 \%)$ \\
\hline Non-ST segment elevation ACS & $729(55.2 \%)$ & $290(68.8 \%)$ \\
\hline Killip Class II-IV ${ }^{\dagger}$ & $254(19.2 \%)$ & $141(33.3 \%)$ \\
\hline $\mathrm{eGFR}<60 \mathrm{~mL} / \mathrm{min} / 1.73 \mathrm{~m}^{2}$ & $333(25.2 \%)$ & $169(39.9 \%)$ \\
\hline Median GRACE score (interquartile range) ( $^{\ddagger}$ & $137(12-167)$ & $145(119-171)$ \\
\hline \multicolumn{3}{|c|}{$\begin{array}{l}\text { na = not applicable. STEMI = ST segment elevation myocardial infarction. ACS = acute coronary syndrome. } \\
\text { eGFR = estimated glomerular filtration rate. } \\
\text { * Values are number of patients (\%) unless otherwise indicated. } \\
\text { † Killip class (scale I-IV) is a risk stratification tool for patients after myocardial infarction; a low Killip class } \\
\text { indicates a lower likelihood of death within the first } 30 \text { days than a high Killip class. } \\
\text { † GRACE = Global Registry of Acute Coronary Events risk score. }\end{array}$} \\
\hline
\end{tabular}

agent(s) (Box 1). Patients with diabetes were older and more likely than non-diabetic patients to have a prior history of hypertension, dyslipidaemia, atrial fibrillation, established atherosclerotic vascular disease, and previous coronary artery revascularisation, but were less likely to be current smokers and to present initially with STEMI. During hospitalisation, those with diabetes were more likely to have Killip Class II-IV heart failure, renal impairment, and a higher GRACE risk score.

\section{Guideline-recommended therapies and invasive management}

Patients with diabetes were less likely than non-diabetic patients to be taking aspirin, clopidogrel, a statin or a $\beta$-blocker at hospital discharge, but were more likely to be taking an ACE-inhibitor or AR-antagonist (Box 2). Patients with diabetes were less likely to undergo early angiography than non-diabetic patients $(67.6 \%$ versus
$82.1 \%$ ), and had a lower overall rate of any coronary revascularisation (PCI/CABG) (46.6\% versus $61.3 \%$ ).

\section{Determinants of long-term outcomes}

After 12 months, patients with diabetes had about twice the mortality rate of non-diabetic patients ( $16.3 \%$ versus $7.3 \%$ ), and also about twice the rate of combined death, non-fatal re-MI or stroke $(28.4 \%$ versus 16.4\%) (Box 2).

Twelve-month survival curves by type of diabetes treatment are shown in Box 3. All groups of diabetic patients, regardless of therapy type, had worse survival than nondiabetic patients (log-rank $P<0.001)$.

Compared with non-diabetic patients, those with diabetes had an $80 \%$ higher adjusted hazard of 12-month mortality (HR, 1.79; 95\% CI, 1.18-2.72; P=0.007), with the highest hazard in those requiring insulin therapy (HR, 2.53; 95\% CI, 1.50-4.28; $P=$ 0.005 ) (Box 4). Invasive management and 


\section{Use of clinical guideline-recommended treatments and 12-month clinical outcomes, by diabetes history at admission*}

\begin{tabular}{lcc} 
Outcome & No diabetes $(n=1321)$ & Diabetes $(n=423)$ \\
\hline Drugs prescribed at discharge & $1181(89.4 \%)$ & $355(83.9 \%)$ \\
Aspirin & $932(70.5 \%)$ & $267(63.1 \%)$ \\
Clopidogrel & $1151(87.1 \%)$ & $353(83.4 \%)$ \\
Statin & $976(73.9 \%)$ & $291(68.8 \%)$ \\
$\beta$-Blocker & $973(73.6 \%)$ & $340(80.4 \%)$ \\
ACE inhibitor/AR antagonist & & \\
Procedures & $1085(82.1 \%)$ & $286(67.6 \%)$ \\
Early angiography & $711(53.8 \%)$ & $150(35.5 \%)$ \\
Percutaneous coronary intervention & $99(7.4 \%)$ & $47(11.1 \%)$ \\
Coronary artery bypass grafting & & $69(16.3 \%)$ \\
Clinical outcomes at 12 months & $96(7.3 \%)$ & $112(26.5 \%)$ \\
All deaths & $205(15.5 \%)$ & $120(28.4 \%)$ \\
Death or non-fatal re-Ml & $217(16.4 \%)$ & \\
Death, re-Ml or stroke &
\end{tabular}

$\mathrm{ACE}=$ angiotensin-converting enzyme. $\mathrm{AR}=$ angiotensin receptor. re-MI = recurrent myocardial infarction. * Values are number of patients (\%).

3 Kaplan-Meier survival curves to 12 months, by type of diabetes therapy

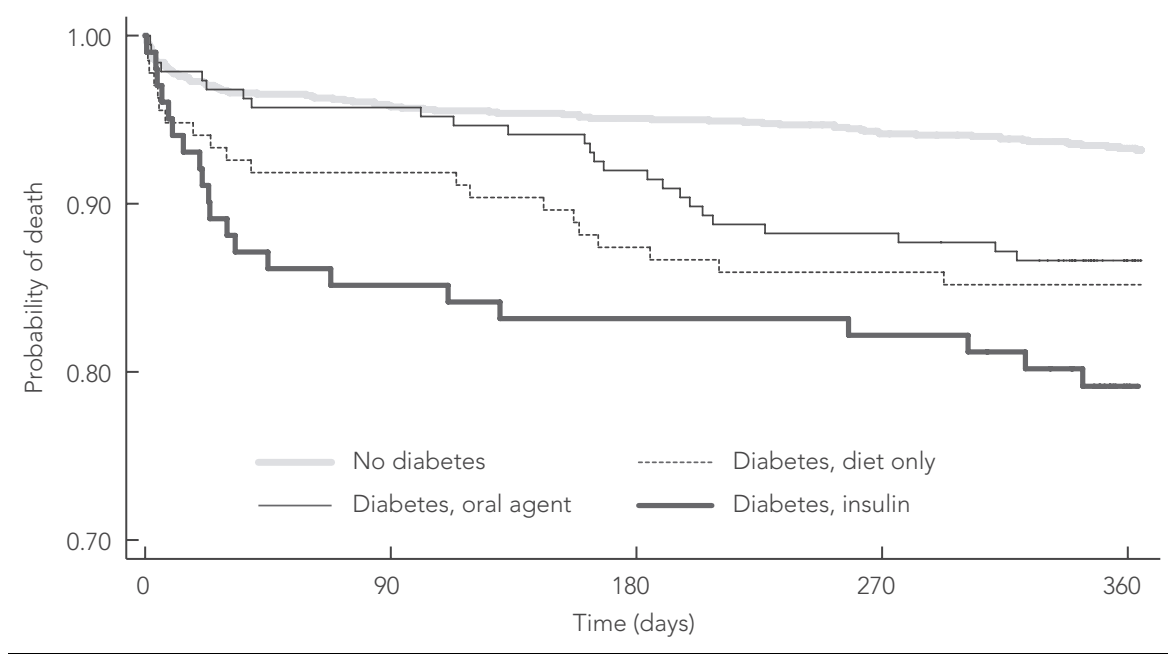

Oral agent $=$ oral hypoglycaemic agent. Log-rank $P$ value $<0.001$.

the prescription at discharge of aspirin, a statin, $\beta$-blocker and/or ACE-inhibitor/ARantagonist were each associated with a significantly reduced hazard of late mortality (Box 4). However, clopidogrel use was associated with an increased mortality hazard. These same treatment covariates, with the exception of a $\beta$-blocker and ACE inhibitor/ AR-antagonist, were also independent predictors of a reduction in combined death, re-MI or stroke (Box 4). When analysis was confined to the subgroup with diabetes, the discharge prescription of a statin (HR, 0.27;
$P<0.001)$, $\beta$-blocker (HR, 0.34; $P<0.001$ ), or ACE-inhibitor/AR-antagonist (HR, 0.30; $P<0.001)$ were each independent predictors of reduced 12-month mortality. Invasive management was also associated with a reduced hazard of death ( $\mathrm{HR}, 0.56 ; 95 \% \mathrm{CI}$, 0.28-1.14), although this did not reach a conventional level of statistical significance $(P=0.11)$. Nevertheless, the observed 5\%$15 \%$ differences in rates of individual therapies, including invasive management, between patients with and without diabetes accounted for little of the excess mortality hazard associated with diabetes, with an HR of 1.84 (95\% CI, 1.29-2.64), decreasing to 1.79 (95\% CI, 1.18-2.72) when the model was adjusted for all therapies.

\section{DISCUSSION}

Our study shows that a substantially higher burden of late morbidity and mortality persists among patients with diabetes who present with acute MI, compared with nondiabetic patients, despite advances in the treatment of ACS. Although much of this excess mortality is explained by diabetes itself, a failure to use evidence-based pharmacotherapies and invasive treatment may also contribute.

A history of diabetes was present in nearly a quarter of patients who presented with acute MI, consistent with results of previous investigations of patients with ACS. ${ }^{2-4,6,7,16}$ Similarly, we found that patients with MI who have diabetes have an inherently higher cardiovascular risk than those without diabetes because of their older age, and higher prevalence of coronary risk factors, established cardiovascular disease and comorbidities, including heart failure and chronic renal impairment. ${ }^{2-4,6,7}$ However, despite controlling for these risk factors and comorbidities in our analysis, diabetes remained a strong independent predictor of late mortality. Although diabetic patients as a group had a worse late clinical outcome, those needing insulin appeared to be at highest risk. This contrasts with a previous investigation, which found that insulin dependence did not portend a worse long-term outcome after controlling for differences in risk characteristics, such as advanced age, longer duration of diabetes and the more severe disease in those who required insulin. ${ }^{17}$

Treatment practices: It has been shown that patients with diabetes and ACS derive even greater benefit than their non-diabetic counterparts from proven risk-modifying therapies. ${ }^{8-10,18}$ Despite this, we found that, within contemporary Australian clinical practice, there remains a disparity in the use of guideline-recommended treatments to the disadvantage of patients with diabetes (Box 2). As a group, diabetic patients at discharge were less likely than nondiabetic patients to be receiving long-term treatment with aspirin, clopidogrel, a statin or a $\beta$-blocker, although they were more likely to be receiving an ACE-inhibitor or AR-antagonist. 
4 Diabetes and other independent predictors of 12-month all-cause mortality and death, myocardial infarction and stroke events

\begin{tabular}{|c|c|c|c|c|}
\hline \multirow[b]{2}{*}{ Variable } & \multicolumn{2}{|c|}{ All-cause mortality } & \multicolumn{2}{|c|}{ Death, re-Ml or stroke } \\
\hline & Hazard ratio $(95 \% \mathrm{Cl})$ & $P$ & Hazard ratio $(95 \% \mathrm{Cl})$ & $P$ \\
\hline Age, per decade $>65$ years & $1.01(1.00-1.02)$ & 0.03 & $1.01(1.00-1.01)$ & ns \\
\hline Prior myocardial infarction & $1.56(1.10-2.20)$ & 0.01 & $1.83(1.43-2.30)$ & $<0.001$ \\
\hline Killip Class & $1.42(1.10-1.82)$ & 0.006 & $1.45(1.23-1.74)$ & $<0.001$ \\
\hline \multicolumn{5}{|l|}{ GRACE score } \\
\hline$<100$ & 1.00 & & 1.00 & \\
\hline $101-150$ & $1.37(0.37-5.06)$ & ns & $1.16(0.67-2.04)$ & ns \\
\hline $151-200$ & $3.43(0.74-15.93)$ & 0.11 & $1.32(0.70-2.50)$ & ns \\
\hline$>200$ & $6.08(1.15-32.24)$ & 0.03 & $1.61(0.75-3.46)$ & ns \\
\hline $\mathrm{eGFR}<60 \mathrm{~mL} / \mathrm{min} / 1.73 \mathrm{~m}^{2}$ & $1.26(0.82-1.94)$ & ns & $1.35(1.07-1.72)$ & 0.01 \\
\hline Dialysis & $2.77(1.32-5.79)$ & 0.007 & $3.35(2.23-5.03)$ & $<0.001$ \\
\hline Angiogram & $0.56(0.38-0.83)$ & 0.004 & $0.68(0.51-0.90)$ & 0.007 \\
\hline \multicolumn{5}{|l|}{ Discharge medications } \\
\hline Aspirin & $0.58(0.39-0.86)$ & 0.006 & $0.59(0.43-0.82)$ & 0.002 \\
\hline Statin & $0.29(0.19-0.43)$ & $<0.001$ & $0.48(0.35-0.65)$ & $<0.001$ \\
\hline$\beta$-Blocker & $0.57(0.41-0.79)$ & 0.001 & $0.75(0.55-1.04)$ & ns \\
\hline ACE inhibitor/AR antagonist & $0.49(0.34-0.71)$ & $<0.001$ & $0.80(0.59-1.04)$ & ns \\
\hline Clopidogrel & $1.90(1.29-2.84)$ & 0.001 & $1.45(1.15-1.83)$ & 0.001 \\
\hline No history of diabetes & 1.00 & & 1.00 & \\
\hline Diabetes present & $1.79(1.18-2.72)$ & 0.007 & $1.44(1.11-1.86)$ & 0.005 \\
\hline No history of diabetes* & 1.00 & & 1.00 & \\
\hline Dietary treatment only & $1.27(0.69-2.34)$ & 0.44 & $1.38(0.90-2.12)$ & 0.14 \\
\hline Oral hypoglycaemic agent & $1.89(1.21-2.94)$ & 0.005 & $1.35(0.99-2.58)$ & 0.06 \\
\hline Insulin therapy & $2.53(1.50-4.28)$ & 0.005 & $1.71(1.13-2.58)$ & 0.01 \\
\hline \multicolumn{5}{|c|}{$\begin{array}{l}\text { re-MI = recurrent myocardial infarction. } n \text { s = not significant. } \\
\text { GRACE = Global Registry of Acute Coronary Events. eGFR = estimated glomerular filtration rate. } \\
A C E=\text { angiotensin-converting enzyme. } A R=\text { angiotensin receptor. }\end{array}$} \\
\hline * Conarate modl lincluding all covari & . & & ording to treatment type. & \\
\hline
\end{tabular}

Moreover, analysis showed that the discharge prescription of aspirin, a statin, $\beta$ blocker, or ACE-inhibitor/AR-antagonist were each associated with a significantly reduced hazard of late deaths and serious cardiovascular events, although prescription of clopidogrel was associated with an increased hazard (Box 4). The latter finding could have been the result of unmeasured confounding or reverse causality (ie, patients who are sicker and more likely to die are also more likely to be prescribed treatments), as clinical trial evidence consistently supports a benefit for clopidogrel in the treatment of high-risk patients with ACS, with or without PCI. ${ }^{19-22}$ However, an adverse effect of clopidogrel in some patient subsets cannot be excluded, as suggested for patients with diabetic nephropathy in a post-hoc analysis of the CHARISMA (Clopidogrel for High Atherothrombotic Risk and
Ischemic Stabilization, Management, and Avoidance) trial, which did not include patients with ACS. ${ }^{23}$

A similar underutilisation of evidencebased pharmacotherapies among diabetic patients with ACS has been demonstrated by data from recent international registries. ${ }^{3-7}$ Discordance between the level of risk and intensity of evidence-based treatment in patients with ACS appears to be a widespread problem that will be overcome only with targeted strategies. ${ }^{24}$

Invasive management: Diabetic patients who present with ACS are more likely than nondiabetic patients to have multivessel and more diffuse coronary artery disease, and to derive greater benefit from an early invasive strategy. ${ }^{10,18}$ However, invasive procedures are often reported to be less used in diabetic patients than in non-diabetic patients, despite current trends towards early revascularisation for high-risk patients with ACS. ${ }^{5-7}$ This disparity was also seen in our study, where diabetic patients had a lower rate of early angiography and coronary revascularisation. Similar to the finding reported in the total ACACIA cohort, ${ }^{12}$ our analysis confirms that a relative long-term mortality advantage is associated with the provision of invasive management among high-risk patients with ACS and documented MI.

A limitation of this study was that the diagnosis of diabetes was based on selfreport, and we were unable to evaluate patients with previously unrecognised diabetes. However, the described differences in outcome might have been even more pronounced had we investigated more actively for diabetes. We did not ascertain the adequacy of metabolic control among the diabetic patients studied, but another recent clinical trial failed to show that intensive metabolic control by means of the acute introduction of long-term insulin had a positive effect on mortality and morbidity in patients with diabetes and acute myocardial infarction (DIGAMI 2). ${ }^{25}$ Our results have to be interpreted cautiously as causal associations cannot be proven using observational data. Furthermore, we are unable to account for unmeasured factors that influence the clinical decision not to prescribe certain cardiac medications or undertake angiography, and these unmeasured factors may have had an important effect on late clinical outcomes.

A concerted effort must be made to optimise the application of evidence-based guideline treatments, including an early invasive management strategy in patients with diabetes who develop ACS.

\section{ACKNOWLEDGEMENTS}

The ACACIA study was organised and run by the following people:

Steering committee. Derek P Chew (Flinders University/Flinders Medical Centre), John Amerena (Geelong Hospital), David Brieger (Concord Hospital), Steve Coverdale (Nambour Hospital), Ashish Soman (sanofi-aventis Australia), James Rankin (Royal Perth Hospital).

Data management and analysis. Cardiovascular Outcomes Research (COR): Carolyn Astley, Danni Molloy, Sue Mattchoss, Luan Huynh.

Sponsor: sanofi-aventis Australia.

Investigators. Tan Ren (Canberra Hospital, ACT), David Brieger (Concord Hospital, NSW), M A Fitzpatrick (Nepean Hospital, NSW), Peter Fletcher (John Hunter Hospital, NSW), David Rees (St George Hospital, NSW), Craig Juergens (Liverpool Hospital, NSW), Jonathon Waites (Coffs Harbour Hospital, NSW), Greg Nelson (Royal North Shore Hospital, NSW), Michael Sinclair (Dubbo Base Hospital, NSW), John Amarena (Geelong Cardiology Practice, VIC), Yean Lim (Western Hospital, VIC), Mark Horrigan 
(Austin Health, VIC), Leeanne Grigg (Royal Melbourne Hospital, VIC), David Eccleston (Northern Hospital Clinical Trials Unit, VIC), Greg Szto (Peninsula Private Hospital, VIC), Gishel New (Box Hill Hospital, VIC), Christopher Medley (Wodonga Regional Health Service, VIC), Steven Coverdale (Nambour General Hospital, QLD), Laurie Howes (Gold Coast Hospital, QLD), David Cross (Wesley Hospital, QLD), Paul Garrahy (Princess Alexandra Hospital, QLD), Darren Walters (Prince Charles Hospital, QLD), Spencer Toombes (Toowoomba Health Services, QLD), Prasad Challa (Cairns Base Hospital, QLD), Kumar Gunawardane (Townsville Hospital, QLD), William Parsonage (Royal Brisbane Hospital, QLD), Raj Shetty (Rockhampton Hospital, QLD), Derek Chew (Flinders Medical Centre, SA), Stephen Worthley (Royal Adelaide Hospital, SA), John Horowitz (Queen Elizabeth Hospital, SA), Samuel Varughese (Mt Gambier Hospital, SA), Christopher Zeitz (Port Augusta Hospital, SA), Margaret Arstall (Lyell McEwin Hospital, SA), Jamie Rankin (Royal Perth Hospital, WA), Barry McKeown (Fremantle Hospital, WA), Johan Janssen (Kalgoorlie Regional Hospital, WA), Joseph Hung (Sir Charles Gairdner Hospital, WA), Philip Roberts-Thomson (Royal Hobart Hospital, TAS), Marcus Iton (Darwin Private Hospital, NT), and Alex Brown (Menzies School of Health Research, NT).

\section{COMPETING INTERESTS}

The study was sponsored by sanofi-aventis Australia, manufacturer of Plavix (clopidogrel). Analysis of data and writing of the manuscript was conducted independently of the sponsor. The sponsor has reviewed the manuscript.

Joseph Hung received sponsorship from sanofiaventis Australia to attend the European Society of Cardiology (ESC) meeting 2008. David Brieger was provided with an unconditional grant from sanofiaventis to run the CONCORDANCE registry, which is not related to this project, and assistance from sanofiaventis to attend American College of Cardiology (ACC) and ESC meetings. John Amerena has sat on advisory boards and given educational lectures for sanofi-aventis and received sponsorship to attend the ACC meeting 2009. Steven Coverdale received an honorarium for participating in the Steering Committee of the ACACIA Registry and for presentation of data. Ashish Soman is an employee of sanofiaventis Australia and New Zealand. Derek Chew has received speaker honoraria and travel assistance from sanofi-aventis Australia.

\section{AUTHOR DETAILS}

Joseph Hung, FRACP, FACC, FCSANZ, Professor of Cardiology ${ }^{1}$

David B Brieger, PhD, FRACP, FCSANZ, Senior Specialist ${ }^{2}$

John V Amerena, FRACP, FACC, FCSANZ,

Director of Cardiology Research ${ }^{3}$

Steven G Coverdale, MB ChB, FRACP, Head of School $^{4}$

James M Rankin, MBBS, FRACP, Cardiologist ${ }^{5}$ Carolyn M Astley, RN, BN(Hons), Operations

Manager, Cardiovascular Outcomes Research ${ }^{6}$

Ashish Soman, MBBS, MRCP(UK), Medical

Affairs Manager ${ }^{7}$

Derek P Chew, MBBS, MPH, FRACP, Professor of Cardiology ${ }^{6}$
1 University of Western Australia School of Medicine and Pharmacology, Sir Charles Gairdner Hospital, Perth, WA.

2 Concord Hospital, Sydney, NSW.

3 University of Melbourne Department of Clinical and Biomedical Science, Geelong Hospital, Geelong, VIC.

4 University of Queensland Sunshine Coast Clinical School, Nambour Hospital, Nambour, QLD.

5 Royal Perth Hospital, Perth, WA.

6 Flinders Medical Centre, Adelaide, SA.

7 sanofi-aventis Australia, Sydney, NSW.

Correspondence: jhung@cyllene.uwa.edu.au

\section{REFERENCES}

1 McGuire DK, Emanuelsson H, Granger CB, et al. Influence of diabetes mellitus on clinical outcomes across the spectrum of acute coronary syndromes. Findings from the GUSTO-llb Study. Eur Heart J 2000; 21: 1750-1758.

2 Malmberg K, Yusuf S, Gerstein HC, et al. Impact of diabetes on long-term prognosis in patients with unstable angina and non-Q-wave myocardial infarction: results of the OASIS (Organization to Assess Strategies for Ischemic Syndromes) Registry. Circulation 2000; 102: 1014-1019.

3 Gitt AK, Schiele R, Wienbergen $H$, et al. Intensive treatment of coronary artery disease in diabetic patients in clinical practice: results of the MITRA study. Acta Diabetologica 2003; 40 Suppl 2: S343S347.

4 Franklin K, Goldberg RJ, Spencer F, et al. Implications of diabetes in patients with acute coronary syndromes. The Global Registry of Acute Coronary Events. Arch Intern Med 2004; 164: 14571463.

5 Brogan GX Jr, Peterson ED, Mulgund J, et al. Treatment disparities in the care of patients with and without diabetes presenting with non-STsegment elevation acute coronary syndromes. Diabetes Care 2006; 29: 9-14.

6 Norhammar A, Lindback J, Ryden L, et al. Improved but still high short- and long-term mortality rates after myocardial infarction in patients with diabetes mellitus: a time-trend report from the Swedish Register of Information and Knowledge about Swedish Heart Intensive Care Admission. Heart 2007; 93: 1577-1583.

7 Donahoe SM, Stewart GC, McCabe CH, et al. Diabetes and mortality following acute coronary syndromes. JAMA 2007; 298: 765-775.

8 Acute Coronary Syndrome Guidelines Working Group. Guidelines for the management of acute coronary syndromes 2006. Med J Aust 2006; 184 (8 Suppl): S1-S32. http://www.mja.com.au/public/ issues/184_08_170406/suppl_170406_fm.html

9 Anderson JL, Adams CD, Antman EM, et al. ACC/AHA 2007 guidelines for the management of patients with unstable angina/non-ST-elevation myocardial infarction: a report of the American College of Cardiology/American Heart Association Task Force on Practice Guidelines (Writing Committee to Revise the 2002 Guidelines for the Management of Patients With Unstable Angina/Non-ST-Elevation Myocardial Infarction) developed in collaboration with the American College of Emergency Physicians, the Society for Cardiovascular Angiography and Interventions, and the Society of Thoracic Surgeons endorsed by the American Association of Cardiovascular and Pulmonary Rehabilitation and the Society for Academic Emergency Medicine. J Am Coll Cardiol 2007; 50: e1-e157.

10 Ryden L, Standl E, Bartnik M, et al. CME guidelines on diabetes, pre-diabetes, and cardiovascu- lar diseases full text: The Task Force on Diabetes and Cardiovascular Diseases of the European Society of Cardiology (ESC) and of the European Association for the Study of Diabetes (EASD). Eur Heart J Suppl 2007; 9 Suppl C: C3-C74.

11 Chew DP, Amerena J, Coverdale S, et al. Current management of acute coronary syndromes in Australia: observations from the acute coronary syndromes prospective audit. Intern Med J 2007; 37: 741-748.

12 Chew DP, Amerena JV, Coverdale SG, et al. Invasive management and late clinical outcomes in contemporary Australian management of acute coronary syndromes: observations from the ACACIA registry. Med J Aust 2008; 188: 691-697.

13 Chew DP, Allan RM, Aroney CN, Sheerin NJ. National data elements for the clinical management of acute coronary syndromes. Med J Aust 2005; 182 (9 Suppl): S1-S16.

14 Mathew TH, Johnson DW, Jones GR; Australasian Creatinine Consensus Working Group. Chronic kidney disease and automatic reporting of estimated glomerular filtration rate: revised recommendations. Med J Aust 2007; 187: 459-463.

15 Tang EW, Wong CK, Herbison P. Global Registry of Acute Coronary Events (GRACE) hospital discharge risk score accurately predicts long-term mortality post acute coronary syndrome. Am Heart J 2007; 153: 29-35.

16 Chih S, McQuillan BM, Kaye J, et al. Abnormal glucose regulation in an Australian acute coronary syndrome population: a prospective study. Diabetes Res Clin Pract 2008; 81: 303-309.

17 Gustafsson I, Hildebrandt P, Seibaek M, et al. Long-term prognosis of diabetic patients with myocardial infarction: relation to antidiabetic treatment regimen. The TRACE Study Group. Eur Heart J 2000; 21: 1937-1943.

18 Roffi M. Early invasive strategy in the diabetic patient with non-ST-segment elevation acute coronary syndromes. Eur Heart J Suppl 2005; 7 Suppl K: K19-K22.

19 CURE Study Investigators. Effects of clopidogrel in addition to aspirin in patients with non-ST segment elevation acute coronary syndromes. $N$ Engl J Med 2001; 345: 494-502.

20 Mehta SR, Yusuf S, Peters RJ, et al. Effects of pretreatment with clopidogrel and aspirin followed by long-term therapy in patients undergoing percutaneous coronary intervention: the $\mathrm{PCl}$ CURE study. Lancet 2001; 358: 527-533.

21 Chen ZM, Jiang LX, Chen YP, et al. Addition of clopidogrel to aspirin in 45,852 patients with acute myocardial infarction: randomised placebo-controlled trial. Lancet 2005; 366: $1607-$ 1621.

22 Sabatine MS, Cannon CP, Gibson CM, et al. Effect of clopidogrel pretreatment before percutaneous coronary intervention in patients with ST-elevation myocardial infarction treated with fibrinolytics: the PCI-CLARITY study. JAMA 2005; 294: 1224-1232.

23 Dasgupta A, Steinhubl SR, Bhatt DL, et al. Clinical outcomes of patients with diabetic nephropathy randomized to clopidogrel plus aspirin versus aspirin alone (a post hoc analysis of the Clopidogrel for High Atherothrombotic Risk and Ischemic Stabilization, Management, and Avoidance [CHARISMA] Trial). Am J Cardiol 2009; 103: 1359-1363.

24 Scott IA, Derhy PH, O'Kane D, et al. Discordance between level of risk and intensity of evidencebased treatment in patients with acute coronary syndromes. Med J Aust 2007; 187: 153-159.

25 Malmberg K, Ryden L, Wedel H, et al. Intense metabolic control by means of insulin in patients with diabetes mellitus and acute myocardial infarction (DIGAMI 2): effects on mortality and morbidity. Eur Heart J 2005; 26: 650-661.

(Received 24 Mar 2009, accepted 24 Aug 2009) 